\title{
Characterization of fast-twitch and slow-twitch skeletal muscles of calsequestrin 2 (CASQ2)-knock out mice: unexpected adaptive changes of fast-twitch muscles only
}

\author{
Giorgia Valle $^{1} \cdot$ Barbara Vergani $^{2} \cdot$ Roberta Sacchetto $^{3} \cdot$ Carlo Reggiani $^{1} \cdot$ \\ Edith De Rosa $^{1} \cdot$ Lisa Maccatrozzo $^{3} \cdot$ Alessandra Nori $^{1} \cdot$ Antonello Villa $^{2} \cdot$ \\ Pompeo Volpe ${ }^{1}$ (D)
}

Received: 27 June 2016 / Accepted: 22 December 2016

(C) Springer International Publishing Switzerland 2017

\begin{abstract}
This study investigates the functional role of calsequestrin 2 (CASQ2) in both fast-twitch and slowtwitch skeletal muscles by using CASQ2-/- mice; CASQ2 is expressed throughout life in slow-twitch muscles, but only in the developmental and neonatal stages in fast-twitch muscles. CASQ2-/- causes increase in calsequestrin 1 (CASQ1) expression, but without functional changes in both muscle types. CASQ2-/- mice have ultrastructural changes in fast-twitch muscles only, i.e., formation of pentads and stacks in the sarcoplasmic reticulum.
\end{abstract}

Keywords Skeletal muscle - Calsequestrin - Adaptation . Knock-out mice

$\begin{array}{ll}\text { Abbreviations } \\ \text { CRT } & \text { Calreticulin } \\ \text { CASQ1 } & \text { Calsequestrin 1 } \\ \text { CASQ2 } & \text { Calsequestrin 2 } \\ \text { KO } & \text { CASQ2-/- } \\ \text { CPVT } & \begin{array}{l}\text { Catecholaminergic polymorphic ventricular } \\ \text { tachycardia }\end{array} \\ \text { ER } & \text { Endoplasmic reticulum } \\ \text { ECCE } & \text { Excitation-coupled calcium entry } \\ \text { EDL } & \text { Extensor digitorum longus }\end{array}$

Pompeo Volpe

pompeo.volpe@unipd.it

1 Dipartimento di Scienze Biomediche dell'Università di Padova, Istituto Interuniversitario di Miologia, Viale G. Colombo 3, 35121 Padova, Italy

2 Consorzio MIA (Microscopy Image Analysis), Università di Milano-Bicocca, 20052 Monza, Italy

3 Dipartimento di Biomedicina Comparata ed Alimentazione dell’Università di Padova, Padova, Italy

$\begin{array}{ll}\text { FDB } & \text { Flexor digitorum longus } \\ \text { GRP78 } & \text { Glucose related protein 78 } \\ \text { GRP94 } & \text { Glucose-related protein 94 } \\ \text { LSR } & \text { Longitudinal SR } \\ \text { SR } & \text { Sarcoplasmic reticulum } \\ \text { SOL } & \text { Soleus } \\ \text { TC } & \text { Terminal cisternae } \\ \text { T-tubules } & \text { Transverse tubules } \\ \text { WT } & \text { Wild type } \\ \text { DD } & \text { Days }\end{array}$

\section{Introduction}

In adult mammals, all type I skeletal muscle fibers contain calsequestrin 1 (CASQ1) and significant amounts $(<20 \%)$ of calsequestrin 2 (CASQ2), whereas type II muscle fibers contain only CASQ1 (Biral et al. 1992; Sacchetto et al. 1993; Murphy et al. 2009). At birth and during early developmental stages, type I and type II skeletal muscle fibers express both CASQs since CASQ isoform transition is accomplished by post-natal week 2-4 (Franzini-Armstrong 1991; Arai et al. 1992; Sacchetto et al. 1993; Franzini-Armstrong and Jorgensen 1994).

CASQ2 is expressed in larger amounts at birth as compared to the adult stage (Sacchetto et al. 1993), is co-expressed in individual muscle fibers with CASQ1 in early developmental stages (Sacchetto et al. 1993), is confined to slow-twitch skeletal muscle fibers in the adult stage (Damiani et al. 1990; Biral et al. 1992; Murphy et al. 2009) and is likewise restricted to the lumen of SR terminal cisternae: very little is known, however, about its specific function during post-natal development and in the adult stage. Transgenic CASQ2-/- (KO) mice (Knollmann et al. 2006; Denegri et al. 2012) have been 
generated as one of the experimental models for recessive catecholaminergic polymorphic ventricular tachycardia (CPVT), an inherited arrhythmogenic disease associated with cardiac arrest in children or young adults without signs of overt skeletal muscle myopathy (Lahat et al. 2001; Knollmann et al. 2006; Song et al. 2007; Raffaele di Barletta et al. 2006; Rizzi et al. 2008; Denegri et al. 2012): their phenotype does not entail any evident change in motor behaviour and mass weight, yet the availability of $\mathrm{KO}$ mice set the stage for investigating the role of CASQ2 in developing and adult skeletal muscles.

A comprehensive investigation at the molecular, functional and ultra-structural level was carried out on predominantly fast- and slow-twitch skeletal muscles, i.e., extensor digitorum longus (EDL) and soleus (SOL), from wild type (WT) and KO mice. Quite unexpectedly, ultrastructural changes took place in fast-twitch skeletal muscle only, i.e., in muscles expressing CASQ2 mainly and only at birth. The present results indicate that CASQ2 exerts a positive and long lasting morphogenetic effect in the early post-natal period and that search for subtle signs of skeletal muscle myopathy may be part of the medical evaluation of recessive CPVT patients.

\section{Materials and methods}

\section{Animal models}

Transgenic homozygous $\mathrm{KO}$ and control C57BL6 WT male mice were previously described (Denegri et al. 2012). All animal experimental protocols were approved by the Animal Care and Use Committee of University of Padova.

\section{RT-PCR}

In RT-PCR experiments, calculations were made using the Applied Biosystems software based upon threshold $(\mathrm{Ct})$ values. $\mathrm{Ct}$ is the fractional cycle number at which the fluorescence passes the fixed threshold. Relative gene expression was quantified as follows: fold change $=2^{-(\mathrm{Ct})}$ where $\mathrm{Ct}=\mathrm{Ct}_{\text {target }}-\mathrm{Ct}_{\text {reference }}$ and $(\mathrm{Ct})=\mathrm{Ct}_{\text {sample }}-\mathrm{Ct}_{\text {control }}$. All values were normalized relative to the expression of beta2 microglobulin and are expressed as mean value \pm SD. All samples were run in triplicate. Comparisons between means of two groups were performed by unpaired two-tales Student's $t$-test. Differences were considered significant at $* P<0.05$. Graphs and statistical analysis were performed by Origin 8 .

\section{Antibodies}

Source of specific antibodies: anti-CASQ2 and antiCASQ1 from Thermo Scientific; anti-glucose related protein 78 (GRP78), anti-glucose-related protein 94 (GRP94) and calreticulin (CRT) from Abcam; actin from Sigma.

\section{Preparation of pure CASQs}

Rabbit CASQ1 and rat CASQ2 was purified from skeletal muscles and hearts, respectively (Slupsky et al. 1987).

\section{Protein profile of skeletal muscle homogenates and quantitative densitometry}

Skeletal muscles were snap frozen in liquid nitrogen and homogenized in 3\% SDS, $1 \mathrm{mM}$ EGTA with protease inhibitors. Quantitative western blotting was carried out on whole skeletal muscle homogenates from WT and KO mice ( $n=4$ for each group). Equal amounts of muscle homogenates $(5-30 \mu \mathrm{g})$ and increasing quantities of purified rabbit CASQ1 (0-300 ng) or rat CASQ2 (0-100 ng), used for calibration curves, were analyzed by SDS-PAGE, as described in Valle et al. (2014). Following transfer to membranes, immunoblots were revealed with the corresponding CASQ1 or CASQ2 primary antibodies and secondary antibodies conjugated with alkaline phosphatase. Intensity of each band was determined by Scion Image software. Protein-signal densities were normalized to the corresponding total protein content, in particular the band referable to myosin heavy chain determined by Coomassie blue staining. For routine western blots, equal amounts of muscle homogenates $(100 \mu \mathrm{g})$ were analyzed and the protein-signal sensities were normalized to the corresponding actin-signal densities within a linear relationship of antigen concentration versus signal density (Valle et al. 2014). Data were expressed as mean \pm standard error (SE). Comparisons between means of two groups were performed by unpaired two-tales Student's $t$-test. Graphs and statistical analysis were performed by Origin 8 .

\section{Electron microscopy (EM)}

SOL and EDL from WT and KO mice were fixed in 2\% $(\mathrm{v} / \mathrm{v})$ glutaraldehyde in $0.1 \mathrm{M}$ cacodylate buffer, $\mathrm{pH}$ 7.4, and post-fixed with $1 \%(\mathrm{w} / \mathrm{v}) \mathrm{OsO}_{4}$ in $0.1 \mathrm{M}$ cacodylate buffer. The samples were then stained en bloc, dehydrated in ethanol and embedded in EPON 812 (Fluka, Buchs, Switzerland) following standard procedures. Ultrathin sections were examined using a Philips CM10 transmission electron microscope. Artwork was created with Photoshop CS. The analysis was carried out on a total of 36 samples 
and not less than 100 fibers for each muscle type at any given age.

\section{Force and contraction kinetics of isolated intact muscles}

EDL and SOL muscles were dissected from the hind limbs of WT and KO male mice ( 3 months old) in warm oxygenated Krebs solution and mounted between a force transducer (SI-H Force Transducer World Precision Instruments, Inc., Sarasota, FL, USA) and a micromanipulator-controlled shaft in a small chamber where oxygenated Krebs solution was continuously circulated. The temperature was kept constant at $25{ }^{\circ} \mathrm{C}$. The stimulation conditions were optimized, and muscle length was increased until force development during tetanus was maximal. The responses to a single stimulus (twitch) or to a series of stimuli at various rates producing unfused or fused tetani were recorded. Time-to-peak tension, time-to-half relaxation, time-to-base tension, and peak-tension were measured in single twitches. Tension was measured in completely fused maximal tetani of different duration (0.5-2 s) at the peak and just after the last stimulus. Data were expressed as mean \pm SD. For analysis of force and contraction kinetics comparison between the two groups (WT, KO mice) was carried out using the student's T-test.

\section{Results and discussion}

\section{Post-natal transition of CASQ isoforms in SOL and EDL skeletal muscles of the WT mouse}

In WT mice, the protein content of CASQ1 and CASQ2 was assessed at three different stages of post-natal development, i.e., at ages of 2, 4 and 8-weeks. The CASQ1 protein content decreased in both SOL (Fig. 1b, solid line) and EDL (Fig. 1b, dashed line). The amount of CASQ1 was always larger in EDL than in SOL, the ratio being between 3 and 2. The CASQ2 protein content showed a peak in SOL at post-natal week 4 (Fig. 1a, solid line; see also Sacchetto et al. 1993), whereas it drastically decreased in EDL during the observed period (Fig. 1a, dashed line). It appears as though the down-regulation of CASQ2 was delayed by 2 weeks in SOL as compared to EDL and that a critical transition occurs at/around post-natal week 4 leading to minimal expression levels in both muscles. As previously shown (Damiani et al. 1990), predominantly fast-twitch muscles of the rabbit express virtually no CASQ2 in the adult stage, whereas the CASQ2 content in slow-twitch skeletal muscle somehow parallels the percentage of type I fibers (Damiani et al. 1990; Murphy et al. 2009). Figure 1c shows the time-course of total expression of both CASQ isoforms in SOL and EDL: the pattern resembles
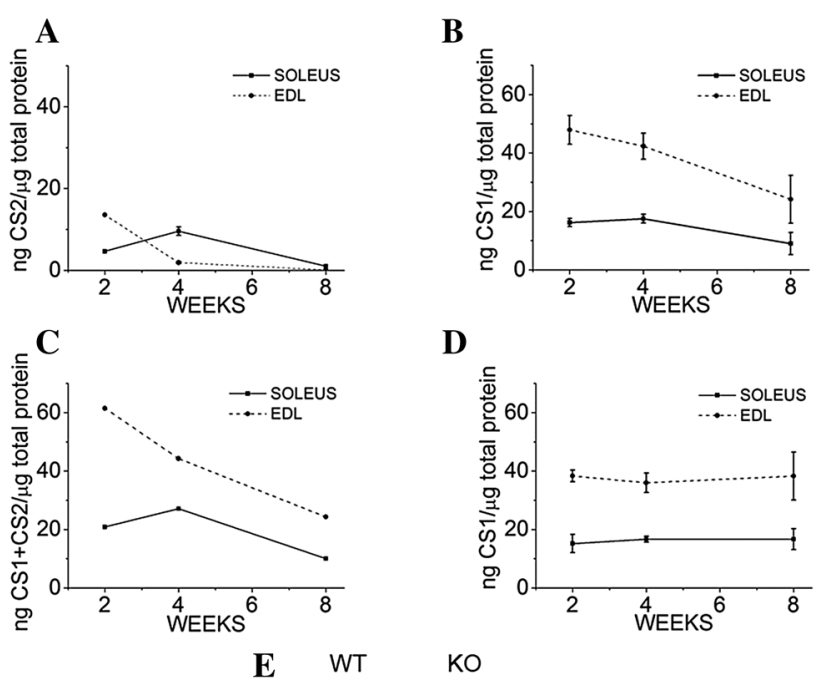

D

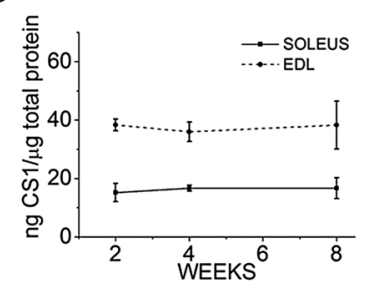

SOLEUS

EDL

Fig. 1 Protein expression in skeletal muscles of WT and KO mice. CASQ2 and CASQ1 protein expression was studied in SOL (solid line) and EDL (dashed line) muscles of WT mice at post-natal week 2, 4 and 8 (a and $\mathbf{b}$, respectively) by Western blotting. In c, CASQ1 and CASQ2 quantities were summed for WT mice. In d, CASQ1 protein expression was analyzed in SOL and EDL muscles from KO mice. Data are given as mean \pm SE for $n=4$. e Shows representative images of CASQ1 expression at post-natal week 8 in SOL and EDL from WT and KO mice, respectively

that of CASQ1 (Fig. 1b), by far the most represented isoform, there is a two-to-three-fold decrease in the amount of CASQ and fast-twitch muscles have a larger amount of CASQ than slow-twitch muscles by a factor of 2.5-3, depending upon age. The latter finding is in agreement with previous quantitative measurements by Leberer and Pette (1986) and Murphy et al. (2009) in rat SOL and EDL, and by Lamboley et al. (2013) in human muscle fibers: the amount of CASQ1 is the major determinant of the maximal SR calcium capacity in fast- and slow-twitch muscle fibers and it determines the maximal (total) calcium content of 4.1 and 1.5 mM in EDL and SOL (cfr. Murphy et al. 2009; Lamboley et al. 2013).

\section{CASQ1 content was increased in skeletal muscles of KO mice}

In $\mathrm{KO}$ mice an interesting phenomenon was observed in both types of skeletal muscle: the protein content of CASQ1 did not change over the time span being analyzed (Fig. 1d, solid and dashed lines for SOL and EDL, respectively). It appears that the CASQ1 content of KO mice is lower at post-natal week 2 but higher at post-natal week 8 as compared to WT mice (panels d, b). There is an increase 
of CASQ1 starting earlier in fast- as compared to slowtwitch skeletal muscles, i.e., post-natal week 2 versus postnatal week 4 (Fig. 1d, e).

In summary, lack of CASQ2 resulted in increased CASQ1 expression suggesting an increase of maximal SR calcium storage in skeletal muscles of CASQ2-1- mice. Increase of CASQ1 levels has been also described in several pathological and physiological conditions, e.g., shortterm denervation of rabbit fast-twitch skeletal muscles (Salvatori et al. 1988; see also Lehotský et al. 1993), dystrophic muscles (Ferretti et al. 2009), after endurance and sprint training in rat EDL (Kinnunen and Mänttäri 2012). Thus, compensatory increase of CASQ1 appears not to be a specific adaptive feature of the CASQ2-/- phenotype.

\section{CASQ1 mRNA in WT and KO mice}

Analysis was then carried out at the mRNA level. CASQ1 mRNA expression in WT and KO skeletal muscles during the post-natal development from 2-day-old to 8-weekold mice. From 2- to 9-day-old mice, we collected whole hind-limb muscles whereas we compared SOL and EDL for 2- and 8-week-old mice. In WT skeletal muscles (Fig. 2, white bars), CASQ1 mRNA started raising at day 7 in hindlimb muscles and reached the highest value at 2-week-old in EDL (cfr. Arai et al. 1992). In adult muscles (8-weekold mice), mRNA decreased both in SOL and EDL as compared to the level of 2-week-old mice. In hind-limb

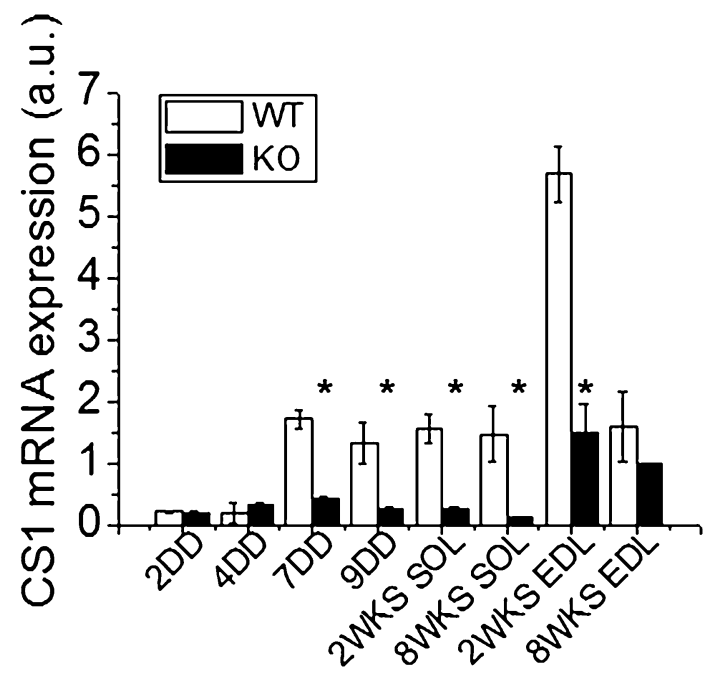

Fig. 2 mRNA expression in skeletal muscles of WT and KO mice. mRNA expression of CASQ1 in skeletal muscles of WT (white columns) and KO (black columns) mice ad different ages. At post-natal days 2, 4, 7 and 9 we studied CASQ1 mRNA expression in hind-limb muscles whereas at post-natal 2 and 8 weeks in two different skeletal muscle, SOL and EDL. Data are given as mean \pm SE for $n=4$. Values of KO samples are different from those of WT samples with $* P<0.05$ muscles from KO mice (Fig. 2, black bars), CASQ1 mRNA spike was absent in 7-day-old mice. SOL from KO mice had no increased mRNA levels whereas EDL displayed a blunted spike in 2-week-old mice. Overall decreased levels of CASQ1 mRNA were detected in KO muscles, although data were significantly different from WT, in some cases only (see* in Fig. 2).

Transcriptional activity is decreased in KO muscles although CASQ1 protein content in adult stages appears to be increased. There appears to be an increase of CASQ1 protein levels despite reduced transcriptional activity that indicates a post-transcriptional regulation of CASQ1.

\section{Ultrastructural analysis of EDL and SOL during post-natal development of $\mathrm{KO}$ mice}

A large wealth of experimental data indicates that postnatal rearrangement of the sarcotubular system, proliferation of terminal cisternae (TC) and triad formation (Franzini-Armstrong and Jorgensen 1994), is associated with up-regulation of specific SR protein and isoform transition, namely CASQ2/CASQ1, at critical ages (Sacchetto et al. 1993, and references therein). Defined temporal patterns of post-natal development have been described in mammalian species, such as mouse and rabbit (Franzini-Armstrong 1991; Villa et al. 1993), before attainment of the adult type organization at about 2-4 weeks after birth, i.e., TC in register with the A-I boundaries and longitudinal SR at the A band level (Franzini-Armstrong 1991). Since CASQ isoform transition occurs in parallel with post-natal morphological changes, we wondered whether the absence of CASQ2, before and after birth, as well as the apparent increase of CASQ1 content (see Fig. 1d), might interfere

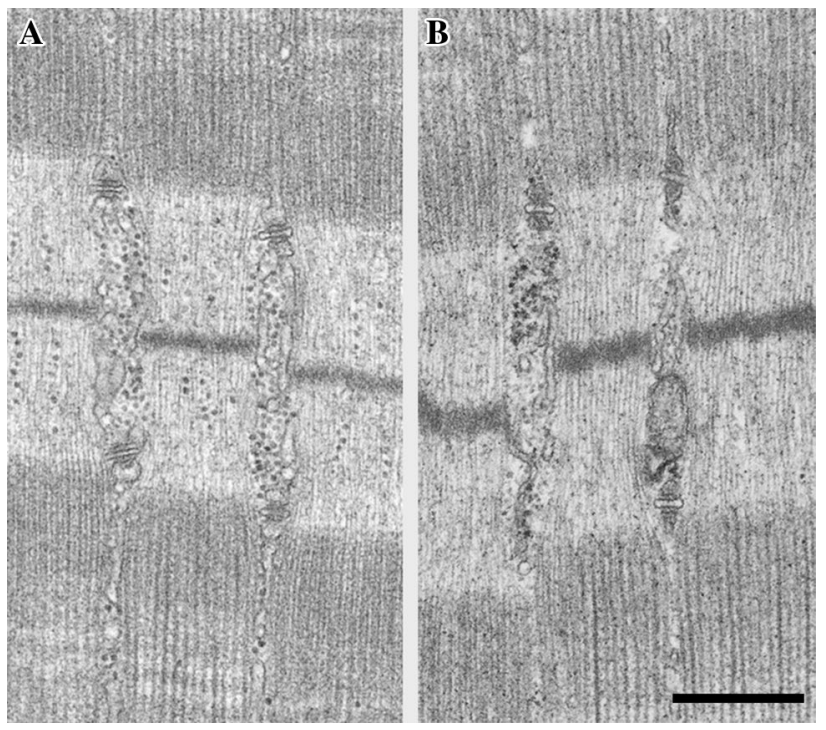

Fig. 3 Electron microscopy of thin sections of EDL and SOL from KO mice at post-natal week 2. a EDL, b, SOL. Bar $500 \mathrm{~nm}$ 
with normal morphological development. Thus, an electron microscope investigation was carried out on SOL and EDL from both WT and KO mice, at post-natal week 2, 4 and 8 (see Figs. 3, 4, 5, 6).

The overall architecture of WT and KO muscles was rather homogeneous and did not show striking differences. At post-natal week 2, both EDL and SOL of KO mice showed well organized SR and sarcomeres (Fig. 3, panels $a$ and $b$, respectively) comparable to those of WT mice (not shown).

Only in some fibers belonging to EDL of KO adult mice, longitudinally-oriented triads and pentads were observed; such structures are usually found in developing normal muscles; in fact, in WT EDL at post-natal week 2, a pentad and a longitudinal triad were observed in panels $a$ and $b$ of Fig. 4, respectively. In KO EDL of paired age, several pentads were observed (panels d-f, Fig. 4), the one in panel $\mathrm{d}$ being particularly complex whereas in panel $\mathrm{c}$ there are longitudinally-oriented triads. Such structures were virtually absent in adult WT EDL but in KO EDL could be observed at post-natal week 4 (panel g) as well as postnatal week 8 (panels $\mathrm{k}$ and $\mathrm{l}$ ). At the latter age, tortuous and variably oriented transverse tubules (T-tubules) were observed in KO EDL (panels h-j, Fig. 4) including pentads.

No such alterations were observed in KO SOL (see below). Thus, CASQ2 might play a morphogenetic role only in fast-twitch muscle fibers belonging to EDL.

EDL from KO mice also displayed differences at the LSR level (Fig. 5). Stacks were observed in many 8-weekold mice (panels a-e) and in about half of the 4 week-old mice (panels $\mathrm{f}, \mathrm{g}$ ), but only in a limited number of fibers, ranging from 10 to $5 \%$, respectively. Stacks are defined as parallel, flat tubules of LSR linked by electron dense material (bridges) having periodicity and width distinct from those shown by "feet" structures at the triad junction (compare LSR stacks and triads, in panel $\mathrm{f}$ and legend to Fig. 5
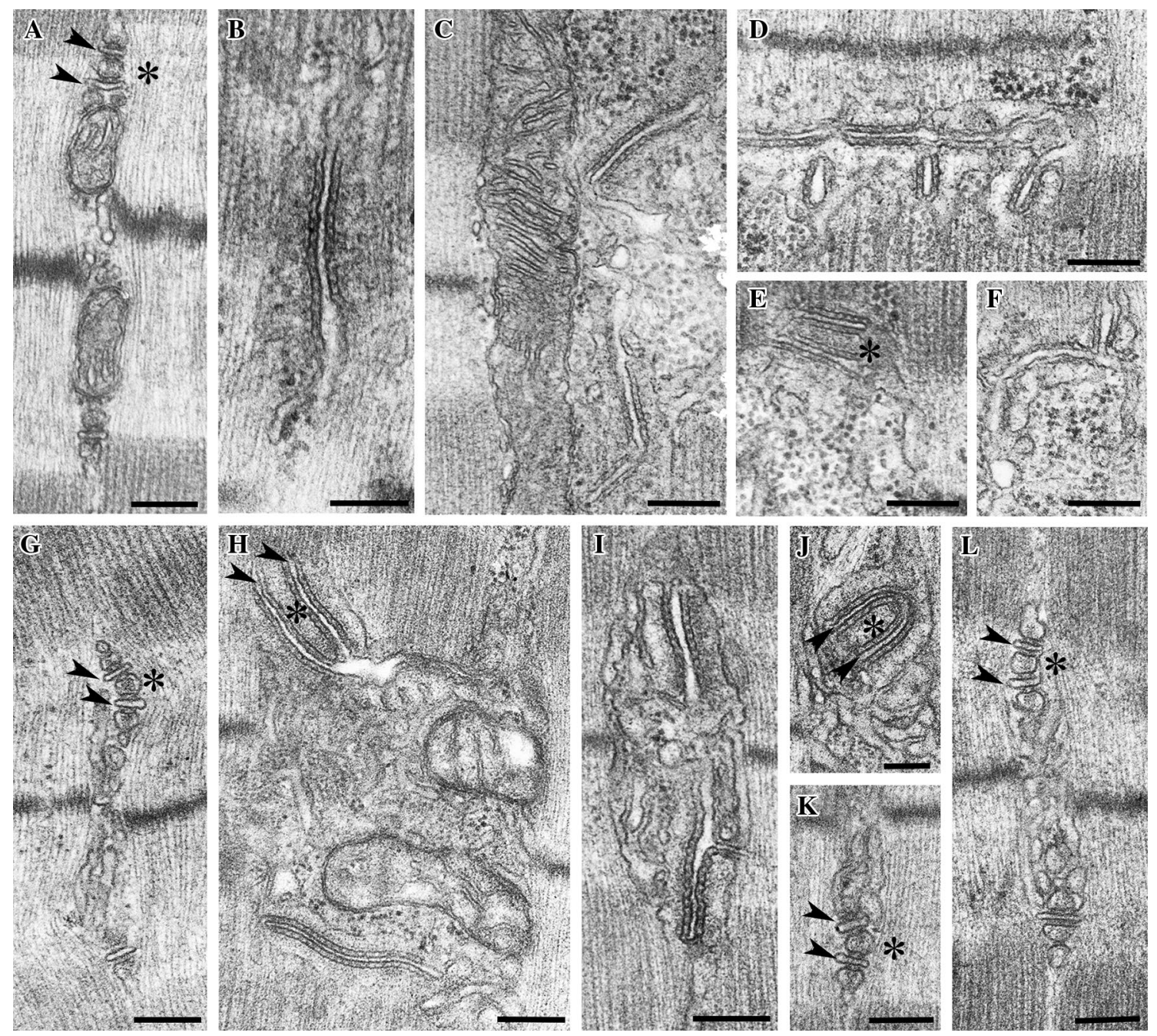

Fig. 4 Electron microscopy of thin sections of EDL from WT and KO mice at post-natal week 2, 4 and 8. a, b WT EDL at post-natal week 2; c-f KO EDL at post-natal week 2; g KO EDL at post-natal

week 4; h-l KO EDL at post-natal week 8. Asterisk pentads, arrowhead T-tubule. Bar $250 \mathrm{~nm}$ in all panels except in $\mathbf{b}, \mathbf{g}, \mathbf{k}$ and $\mathbf{l}$ where is $200 \mathrm{~nm}$ 
Fig. 5 Electron microscopy of thin sections of EDL from KO mice at post-natal week 4 and 8. a-e KO EDL at postnatal week 8; f, g KO EDL at post-natal week 4 . Square bracket stacks, black arrow triadic feet, white arrow stack bridges. Measurements of width and periodicity of triadic feet and stack bridges are the following and are expressed in $\mathrm{nm} \pm$ SD: feet, width $20.5 \pm 2.6$ for $\mathrm{n}=50$; periodicity $27.5 \pm 1.8$ for $\mathrm{n}=120$; bridges, width $17.3 \pm 1.8$ for $\mathrm{n}=50$; periodicity $11.4 \pm 1.8$ for $\mathrm{n}=72$. Bar is $200 \mathrm{~nm}$ in all panels except in c where is $100 \mathrm{~nm}$
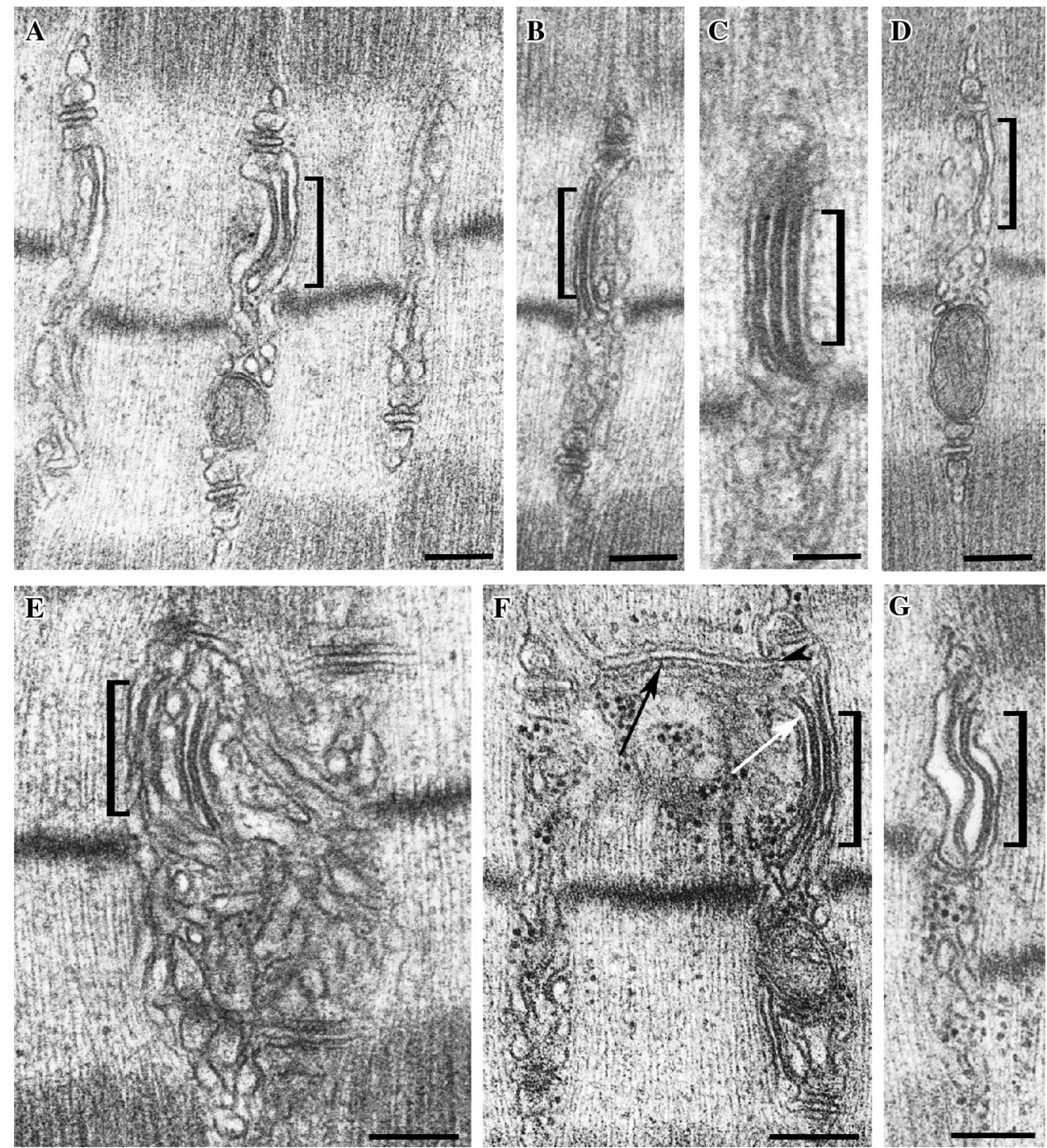

for measurements of width and periodicity). Often there were three or more longitudinal tubules (panels c-f) disposed in half of the I band and continuous with SR. Sometime, when the section plan is appropriate, stack-forming tubules merged and continued into a terminal cisterna (panels $a, f$ ). If present, stacks were observed along the entire long axis of the fiber.

Figure 6 shows pictures of SOL at different ages from both WT and KO mice. SOL from KO mice, at all ages being analyzed, did not display any of the changes described in EDL from KO mice. Muscle fibers of mouse SOL belong in approximately equal ratio to type I and type IIA (Pellegrino et al. 2003). The random choice of fibers under the electron microscope guarantees that fibers of both types have been observed and that in none of them, either fast(IIA)- or slow(I)-twitch fibers, alterations, such as pentads, stacks, etc., were found.

Quite unexpectedly, thus, morphological changes were observed only in EDL from KO mice and typical structures are defined as pentads and stacks. Pentads and higher order combination of T-tubules and TC are convoluted T-tubules and TC serially juxtaposed (see Fig. 4i and asterisks) and usually localized at the a-i interface (cfr., Paolini et al. 2007); stacks are two or more parallel, flat and bridged tubules of LSR in continuity with SR and localized at the I-band (cfr. Boncompagni et al. 2012). Such changes can be evoked by either lack of CASQ2 and/or by increase of CASQ1.

Stacks and pentads appear to be different both in origin and sarcomere localization. Since only fast-twitch muscles are adapting, it is tempting to speculate that lack of CASQ2 in critical morphogenetic stages determines observed ultrastructural changes rather than increased levels of CASQ1, since no such changes are found in slow-twitch muscles in which CASQ1 was likewise increased.

There are four fiber types of skeletal muscle fibers in mammals identified by the presence of specific myosin heavy chain isoforms: mitochondria-rich slow type I fibers, 

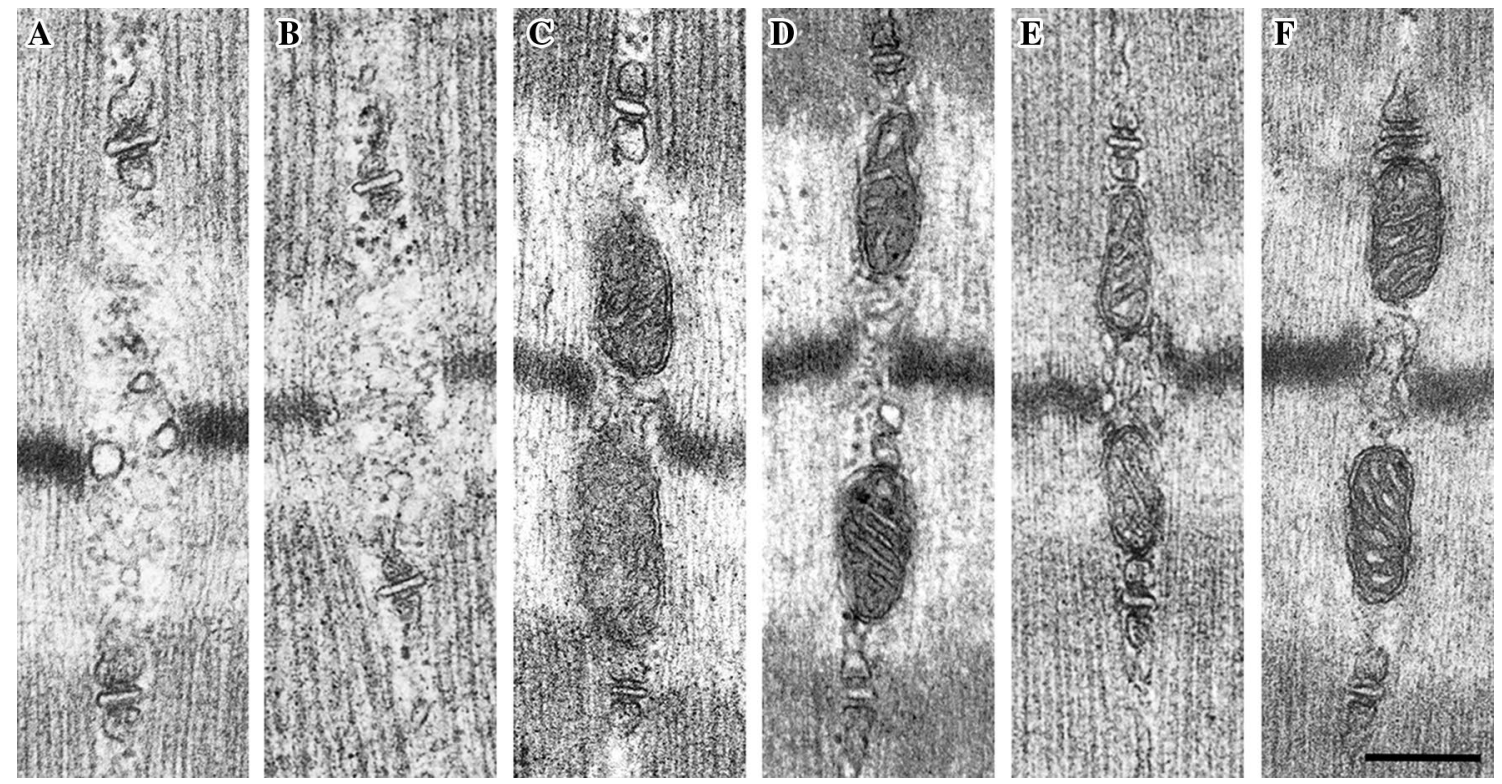

Fig. 6 Electron microscopy of thin sections of SOL from WT and KO mice at post-natal week 2, 4 and 8. a, c, e SOL from WT mice, b, d, $\mathbf{f}$ SOL from KO mice. a, b Post-natal week 2, c, d post-natal week 4, e, f post-natal week 8. Bar $200 \mathrm{~nm}$

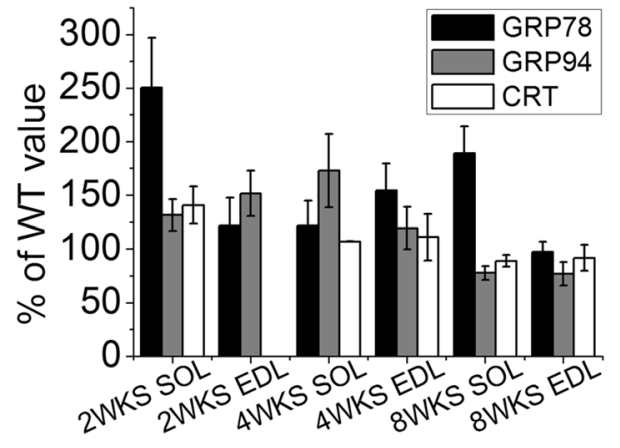

Fig. 7 Markers of ER stress activation. Activation of ER stress was monitored through expression of GRP78 (black columns), GRP94 (gray columns) and CRT (white columns) at post-natal week 2, 4 and 8 in SOL and EDL, from KO and WT mice. Average percentages for $\mathrm{KO}$ mice are given as mean $\pm \mathrm{SE}, \mathrm{n}=4$

mitochondria-rich fast IIA and IIX fibers, and mitochondria-poor fast IIB fibers. Taking into account the fiber type composition of slow-twitch and fast-twitch skeletal muscles in the mouse (Pellegrino et al. 2003), it would appear that only type IIX and type IIB fibers, which are present in EDL and absent in SOL, are sensitive to lack of CASQ2. Stacks and pentads observed in EDL from KO mice are by no means specific of such a phenotype. Proliferation of junctional domains, formation of stacks and of tubular aggregates are described in a number of disparate genetic and acquired conditions (Engel 1994; Salvatori et al. 1988; Woo et al. 2012; Amoasii et al. 2013, and references therein). Pentads and heptads also develop after 1 week of denervation in rat EDL (Takekura et al. 1996) but are also observed in normal swim-bladder muscle of a scorpionfish (Suzuki et al. 2003); stacks, very similar to those described in the present paper, have been reported by Boncompagni et al. (2012) in transgenic mice lacking yet other junctional SR proteins, i.e., triadin and junctin, and by Ko et al. (2011) in doxycycline-induced KO mice lacking junctophilin 1 and junctophilin 2 (see Franzini-Armstrong 2012), as well as following hypoxic injury (Schiaffino 2012). Thus, it appears that stacks and pentads are adaptive yet non-specific responses.

\section{Ablation of CASQ2 does not activate endoplasmic reticulum (ER) stress in skeletal muscles}

Molecular adaptation is occurring in transgenic animals and is a major cornerstone of pathophysiological studies. In particular, ER stress is an adaptive response that does not always take place (Knollmann et al. 2006; Valle et al. 2014). ER stress does not occur in CASQ2-/- cardiomyocytes, whereas it occurs in cardiomyocytes from Knock-in CASQ2 $2^{\mathrm{R} 33 \mathrm{Q}+/+}$ mice (Valle et al. 2014).

We followed the expression of ER stress markers, GRP78 and GRP94, in order to assess if the absence of CASQ2 in skeletal muscles might cause expression imbalance of other proteins associated with CASQ2, from postnatal week 2 to postnatal week 8 . There was a slight but not significant increase of GRP78 both in SOL and EDL (Fig. 7). In addition, we followed the expression of CRT and found no significant increase of the protein in either 
Table 1 Force and kinetic measurements were carried out as described in "Materials and methods" on intact EDL or SOL muscles dissected out from 12-week-old mice

\begin{tabular}{llllll}
\hline & SOL & & & EDL & \\
\cline { 2 - 3 } \cline { 5 - 6 } \cline { 5 - 6 } & WT & KO & & WT & KO \\
\hline Number of samples & 10 & 12 & 9 & 11 \\
Muscle weight $(\mathrm{g})$ & $12.1 \pm 2.0$ & $11.0 \pm 2.2$ & & $12.4 \pm 1.7$ & $12.0 \pm 1.9$ \\
Twitch, time to peak $(\mathrm{ms})$ & $38.6 \pm 8.0$ & $49.7 \pm 12.8 *$ & & $17.9 \pm 2.7$ & $18.8 \pm 7.4$ \\
Twitch relaxation time $(\mathrm{ms})$ & $82.5 \pm 16.2$ & $96.4 \pm 19.5$ & & $33.0 \pm 6.6$ & $32.5 \pm 10.5$ \\
Twitch peak force $\left(\mathrm{mN} / \mathrm{mm}^{2}\right)$ & $22 \pm 11$ & $25 \pm 12$ & & $49 \pm 14$ & $53 \pm 17$ \\
Tetanus, force (mN/mm ${ }^{2}$ ) & $112 \pm 45$ & $143 \pm 58$ & & $188 \pm 46$ & $210 \pm 52$ \\
Twitch/tetanus ratio & $0.194 \pm 0.040$ & $0.175 \pm 0.031$ & & $0.267 \pm 0.027$ & $0.276 \pm 0.017$ \\
Tension decay (residual tension at $3 \mathrm{~s})$ & $0.78 \pm 0.07$ & $0.86 \pm 0.04$ & & $0.59 \pm 0.04$ & $0.49 \pm 0.11$ \\
\hline
\end{tabular}

Data are expressed as mean $\pm \mathrm{SD} ; \mathrm{n}$ was as specified except for tension decay where: SOL WT $\mathrm{n}=4$, SOL $\mathrm{KO} n=4$, EDL WT $n=2$, EDL KO $n=3$. Values of KO samples are different from those of WT samples with $* P<0.05$
SOL or EDL (Fig. 7). These data indicate that during postnatal development there were no signs of ER stress in skeletal muscles of $\mathrm{KO}$ mice, as it happens for cardiomyocytes of the very same model (Valle et al. 2014).

\section{Functional studies: force and kinetic measurements}

In a recent paper (Mosca et al. 2016) resting calcium concentration, averaged peak and half time of the decay of myoplasmic calcium transients, excitation-coupled calcium entry (ECCE) have been measured in single fibers obtained from flexor digitorum longus (FDB) of WT and KO mice. Resting calcium concentration and calcium influx through nifedipine-sensitive voltage dependent calcium channels are not changed in FDB fibers from KO mice. The half time of the decay was significantly prolonged in $\mathrm{KO}$ fibers (Mosca et al. 2016) indicating that myoplasmic calcium concentration is phasically higher for longer time intervals during a single twitch, thus possibly affecting nuclear transcription.

Based on the foregoing, it is possible that force and kinetic measurements would unveil additional differences between KO and WT samples. Force and kinetic measurements (time to peak and relaxation time for twitch and tension decay) on intact SOL and EDL muscles were carried out on specimens from adult WT and KO mice. Of the several parameters measured, as shown in Table 1, no one was significantly altered in either SOL or EDL from KO mice but twitch time to peak of SOL from KO mice $(\mathrm{P}<0.05)$ which showed a significant prolongation.

\section{Conclusion}

The present study indicates that CASQ2 has a long lasting morphogenetic role on its own, distinct from that exerted by CASQ1 (see Paolini et al. 2007), and likely related to its expression before and right after birth, up to post-natal day 7 (cfr. Arai et al. 1992). Myoblasts, myotubes and skeletal muscle fibers from KO mice never go through proper embryonic and, later, neonatal stages since CASQ2 is lacking. The ultrastructural changes evoked by ablation of CASQ2 are displayed only in fast-twitch muscle fibers and are not only different from those evoked by lack of CASQ1 but they are shown in the presence of an increased content of CASQ1.

Acknowledgements The authors thank Silvia G. Priori, Barbara Mosca and Francesco Zorzato for their keen interest in preliminary stages of the work. Funds have been provided by Telethon (Grant GGP11141) and MIUR (Grant 2010BWY8E9) to PV.

\section{References}

Amoasii L, Hnia K, Chicanne G, Brech A, Cowling BS, Müller MM, Schwab Y, Koebel P, Ferry A, Payrastre B, Laporte J (2013) Myotubularin and PtdIns3P remodel the sarcoplasmic reticulum in muscle in vivo. J Cell Sci 126:1806-1819

Arai M, Otsu K, MacLennan DH, Periasamy M (1992) Regulation of sarcoplasmic reticulum gene expression during cardiac and skeletal development. Am J Physiol 262:C614-C620

Biral D, Volpe P, Damiani E, Margreth A (1992) Coexistence of two calsequestrin isoforms in slow-twitch skeletal muscle fibers. FEBS Lett 299:175-178

Boncompagni S, Thomas M, Lopez JR, Allen PD, Yuan Q, Kranias EG, Franzini-Armstrong C, Perez CF (2012) Triadin/Junctin double null mouse reveals a differential role for triadin and junctin in anchoring CASQ to the jSR and regulating $\mathrm{Ca}^{2+}$ homeostasis. PLoS One 7:1-12

Damiani E, Volpe P, Margreth A (1990) Coexpression of two isoforms of calsequestrin in rabbit slow-twitch muscle. J Muscle Res Cell Motil 11:522-530

Denegri M, Cruz JEE, Boncompagni S, De Simone S, Auricchio A, Villani L, Volpe P, Protasi F, Napolitano C, Priori SG (2012) Viral gene transfer rescues arrhythmogenic phenotype and ultrastructural abnormalities in adult calsequestrin-null mice with inherited arrhythmias. Circ Res 110:663-668 
Engel AG (1994) Ultrastructural changes in diseased muscles. In: Engel AG, Franzini-Armstrong C (eds) Myology, 3rd edn. McGraw-Hill, NY, 749-888

Ferretti R, Marques MJ, Pertille A, Santo Neto H (2009) Sarcoplasmic-endoplasmic-reticulum $\mathrm{Ca}^{2+}$-ATPase and calsequestrin are overexpressed in spared intrinsic laryngeal muscles of dystrophin-deficient mdx mice. Muscle Nerve 39:609-615

Franzini-Armstrong C (1991) Simultaneous maturation of transverse tubule and sarcoplasmic reticulum during muscle differentiation in the mouse. Dev Biol 146:353-363

Franzini-Armstrong C (2012) Junctophilins and SR docking in muscle. FASEB J 26:1774

Franzini-Armstrong C, Jorgensen AO (1994) Structure and development of E-C coupling units in skeletal muscle. Annu Rev Physiol 56:509-534

Kinnunen S, Mänttäri S (2012) Specific effects of endurance and sprint training on protein expression of calsequestrin and SERCA in mouse skeletal muscle. J Muscle Res Cell Motil 33:123-130

Knollmann BC, Chopra N, Hlaing T, Akin B, Yang T, Ettensohn K, Knollmann BE, Horton KD, Weissman NJ, Holinstat I, Zhang W, Roden DM, Jones LR, Franzini-Armstrong C, Pfeifer K (2006) Casq2 deletion causes sarcoplasmic reticulum volume increase, premature $\mathrm{Ca}^{2+}$ release, and catecholaminergic polymorphic ventricular tachycardia. J Clin Invest 116:2510-2520

Ko JK, Choi KH, Zhao X, Komazaki S, Pan Z, Weisleder N, Ma J (2011) A versatile single-plasmid system for tissue-specific and inducible control of gene expression in transgenic mice. FASEB J 25:2638-2649

Lahat H, Pras E, Olender T, Avidan N, Ben-Asher E, Man O, LevyNissenbaum E, Khoury A, Lorber A, Goldman B, Lancet D, Eldar M (2001) A missense mutation in a highly conserved region of CASQ2 is associated with autosomal recessive catecholamine-induced polymorphic ventricular tachycardia in Bedouin families from Israel. Am J Hum Genet 69:1378-1384

Lamboley CR, Murphy RM, McKenna MJ, Lamb GD (2013) Endogenous and maximal sarcoplasmic reticulum calcium content and calsequestrin expression in type I and type II human skeletal muscle fibres. J Physiol 591:6053-6068

Leberer E, Pette D (1986) Immunochemical quantification of sarcoplasmic reticulum Ca-ATPase, of calsequestrin and of parvalbumin in rabbit skeletal muscles of defined fiber composition. Eur J Biochem 156:489-496

Lehotský J, Bezáková G, Kaplán P, Raeymaekers L (1993) Distribution of $\mathrm{Ca}^{2+}$ modulating proteins in sarcoplasmic reticulum membranes after denervation. Gen Physiol Biophys 12:339-348

Mosca B, Eckhardt J, Bergamelli L, Treves S, Bongianino R, De Negri M, Priori SG, Protasi F, Zorzato F (2016) Role of the JP45-calsequestrin complex on calcium entry in slow twitch skeletal muscles. J Biol Chem 291: 4555-4565

Murphy RM, Larkins NT, Mollica JP, Beard NA, Lamb GD (2009) Calsequestrin content and SERCA determine normal and maximal Ca2 + storage levels in sarcoplasmic reticulum of fast- and slow-twitch fibres of rat. J Physiol 587:443-460

Paolini C, Quarta M, Nori A, Boncompagni S, Canato M, Volpe P, Allen PD, Reggiani C, Protasi F (2007) Re-organized stores and impaired calcium handling in skeletal muscle of mice lacking calsequestrin-1. J Physiol 583:767-784
Pellegrino MA, Canepari M, Rossi R, D’Antona G, Reggiani C, Bottinelli R (2003) Orthologous myosin isoforms and scaling of shortening velocity with body size in mouse, rat, rabbit and human muscles. J Physiol 546:677-689

Raffaele di Barletta M, Viatcheko-Karpinski S, Nori A, Memmi M, Terentyev D, Turcato F, Valle G, Rizzi N, Napolitano C, Gyorke S, Volpe P, Priori SG (2006) Clinical phenotype and functional characterization of CASQ2 mutations associated with catecholaminergic polymorphic ventricular tachycardia. Circulation 114:1012-1019

Rizzi N, Liu N, Napolitano C, Nori A, Turcato F, Colombi B, Bicciato S, Arcelli D, Spedito A, Scelsi M, Villani L, Esposito G, Boncompagni S, Protasi F, Volpe P, Priori SG (2008) Unexpected structural and functional consequences of the R33Q homozygous mutation in cardiac calsequestrin. A complex arrhythmogenic cascade in a knock in mouse model. Circ Res 103:298-306

Sacchetto R, Volpe P, Damiani E, Margreth A (1993) Postnatal development of rabbit fast-twitch skeletal muscle: accumulation, isoform transition and fiber distribution of calsequestrin. J Muscle Res Cell Motil 14:646-653

Salvatori S, Damiani E, Zorzato F, Volpe P, Pierobon S, Quaglino D, Salviati G, Margreth A (1988) Denervation-induced proliferative changes of triads in rabbit skeletal muscle. Muscle Nerve 11:1246-1259

Schiaffino S (2012) Tubular aggregates in skeletal muscle: just a special type of protein aggregates? Neuromuscul Disord 22:199-207

Slupsky JR, Ohnishi M, Carpenter MR, Reithmeier RA (1987) Characterization of cardiac calsequestrin. Biochemistry 26:6539-6544

Song L, Alcalai R, Arad M, Wolf CM, Toka O, Conner DA, Berul CI, Eldar M, Seidman CE, Seidman JG (2007) Calsequestrin 2 (CASQ2) mutations increase expression of calreticulin and ryanodine receptors, causing catecholaminergic polymorphic ventricular tachycardia. J Clin Invest 117:1814-1823

Suzuki S, Nagayoshi H, Ishino K, Hino N, Sugi H (2003) Ultrastructural organization of the transverse tubules and the sarcoplasmic reticulum in a fish sound-producing muscle. J Electron Microsc (Tokyo) 52:337-347

Takekura H, Kasuga N, Kitada K, Yoshioka T (1996) Morphological changes in the triads and sarcoplasmic reticulum of rat slow and fast muscle fibres following denervation and immobilization. $\mathbf{J}$ Muscle Res Cell Motil 17:391-400

Valle G, Boncompagni S, Sacchetto R, Protasi F, Volpe P (2014) Post-natal adaptation in a knock-in mouse model of calsequestrin 2-linked recessive catecholaminergic polymorphic ventricular tachycardia. Exp Cell Res 321:178-189

Villa A, Podini P, Nori A, Panzeri MC, Martini A, Meldolesi J, Volpe P (1993) The endoplasmic reticulum-sarcoplasmic reticulum connection. II. Postnatal differentiation of the sarcoplasmic reticulum in skeletal muscle fibers. Exp Cell Res 209:140-148

Woo JS, Cho CH, Lee KJ, Kim DH, Ma J, Lee EH (2012) Hypertrophy in skeletal myotubes induced by junctophilin-2 mutant, $\mathrm{Y} 141 \mathrm{H}$, involves an increase in store-operated $\mathrm{Ca}^{2+}$ entry via Orai1. J Biol Chem 287:14336-14348 